\title{
Isolated Superior Mesenteric Vein Thrombosis in Acute Pancreatitis: A Case Report
}

\author{
Alpha Oumar Toure*, Ousmane Ka, Ibrahima Ka, Mamadou Seck, Ousmane Thiam, \\ Mohamadou Lamine Gueye, Ibrahima Konate, Mamadou Cisse, Madieng Dieng, \\ Abdarahmane Dia, Cheikh Tidiane Toure \\ Service de Chirurgie Générale et Digestive, CHU Aristide Le Dantec, Dakar, Sénégal \\ Email: ${ }^{*}$ alpha.oumar@yahoo.fr
}

Received 7 May 2015; accepted 30 June 2015; published 3 July 2015

Copyright (C) 2015 by authors and Scientific Research Publishing Inc. This work is licensed under the Creative Commons Attribution International License (CC BY). http://creativecommons.org/licenses/by/4.0/

\section{Abstract}

The most common vascular complication of acute pancreatitis is thrombosis of the splenic vein. Isolated thrombosis of the superior mesenteric vein is rare and may lead to mesenteric ischemia and bowel infarction. We report the case of a 39 years old patient received for acute pancreatitis with a Ranson score less than 3 and image scanner for a grade $C$ of Balthazar and a superior mesenteric vein thrombosis. The outcome was favorable with improvement of symptoms under medical treatment including anti-coagulant therapy. The contrast enhancing abdominal CT showed an absence of superior mesenteric thrombosis.

\section{Keywords}

Pancreatitis, Superior Mesenteric Vein Thrombosis, Anti-Coagulant

\section{Introduction}

Acute pancreatitis can cause many vascular complications, arterial or venous. The most frequent complications are thrombosis of the splenic or portal vein [1]-[3]. Thrombosis of the superior mesenteric vein without simultaneous thrombosis of the splenic or portal vein is uncommon and is usually seen with pancreatic neoplasms or intra-abdominal sepsis [1]. It is an important clinical entity because of the risk of occurrence of mesenteric ischemia and bowel infarction [1]. We report a case of TSMV discovered by contrast enhancing abdominal CT in a patient managed for acute pancreatitis.

\footnotetext{
"Corresponding author.
} 


\section{Case Presentation}

A 39-year-old woman presented with history of moderately severe, sudden onset of epigastric pain. It was associated with vomiting and constipation. Examination on admission was relevant for epigastric bloating and sensibility. The vital signs including the temperature and pulse were normal.

Hematological and biochemical tests: The initial investigation showed: Hematocrit: 33\%; WBC count:

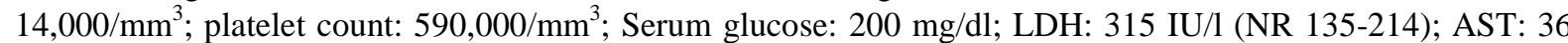
IU/l (normal < 30). The subsequent investigations, 48 hours after admission, showed: Hematocrit rate: 30.5\%;

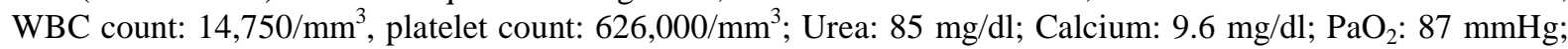
Alkaline reserve was $4.3 \mathrm{~m} \cdot \mathrm{mol} / \mathrm{l}$; Serum Na: $127 \mathrm{~m} \cdot \mathrm{Eq} / \mathrm{l}$; Serum K: $3.4 \mathrm{~m} \cdot \mathrm{Eq} / \mathrm{l}$.

Imaging: The contrast-enhanced CT showed a heterogeneous swollen pancreas associated with a peri-pancreatic collection and fat densification. There was also an absence of opacification of the superior mesenteric vein. The gallbladder was unremarkable. These images corresponded to a grade $\mathrm{C}$ in the classification of Balthazar [4] as seen on Figure 1. The subsequent imaging was also revealed the presence of cholelithiasis.

Management: The patient was managed conservatively with Intravenous fluid followed by oral fluid diet and then eventually changed to usual diet. The initial medication was included tramadol injection $100 \mathrm{mg} / 12 \mathrm{hours}$ for abdominal pain, amoxicillin + clavulanic acid injection (1 g/12hours), ranitidine injection (50 mg/8hours). And enoxaparin $0.4 \mathrm{~g} / 12$ hours subcutaneously.

Five days after admission, the patient complained about persistent epigastric pain with signs of upper GIT bleeding, for that reason we performed gastroduodenoscopy which revealed the presence of hemorrhagic erosive gastritis. Ranitidine was substituted with omeprazole $20 \mathrm{mg} / 12$ hours.

The outcome was favorable. A second contrast-enhanced CT showed good permeability of mesenteric superior vein. She was discharged home 15 days after admission.

\section{Discussion}

Superior mesenteric vein thrombosis is a rare condition with an incidence varying between $0.2 \%$ and $2 \%$ in autopsy series [2]. It is recognized as a clinical entity since 1935, by Warren and Eberhardt [3]. Several risk factors

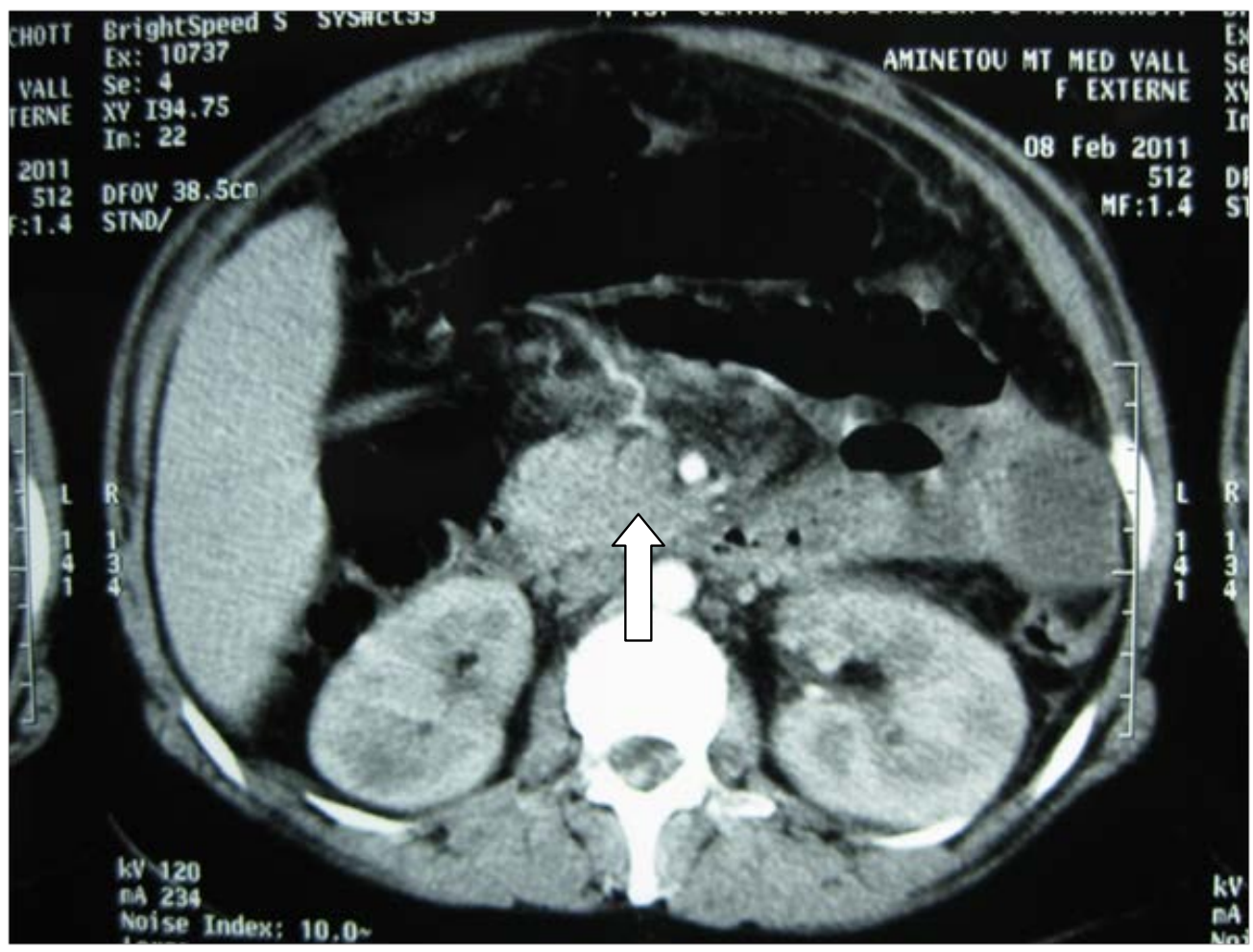

Figure 1. Balthazar C pancreatitis and superior mesenteric vein thrombosis (arrow). 
are associated with the occurrence of TSMV, including pancreatitis, cholecystitis or cholangitis, oral contraceptives, portal hypertension and abdominal trauma [3] [5] [6]. The pathogenesis of venous thrombosis, in case of pancreatitis, could be a venous compression by a pancreatic pseudo-cyst, or an imbalance between coagulation and fibrinolysis because of local inflammatory processes. That results in spasm, and vascular stasis [3] [5] [7].

The TSMV diagnosis is difficult because of its rarity and the lack of symptoms specificity. It usually manifests as a vague abdominal pain that can easily be masked by the pancreatitis as in our patient. Contrastenhanced abdominal CT is the main investigation with sensitivity higher than 90\% [3] [5] [6]. The magnetic resonance imaging and ultrasound are also used, although less commonly. The TSMV is classified into 3 types according to the evolution of pain: acute, sub-acute and chronic. The acute form is at a higher risk of bowel infarction. In these cases, diagnosis and treatment should be early. Initially, they were treated surgically with mortality rates of $34 \%$ and a $5 \%$ survival for patients who did not undergo surgery [3] [5]. Currently, anticoagulation alone, with close monitoring is an acceptable mean of therapy as ischemia of the small bowel has not evolved to necrosis and perforation. Some cases of spontaneous thrombus dissolution have been reported in the literature [1] [5]. The effectiveness of thrombolytic therapy was mentioned by some authors even if the delay in diagnosis makes it obsolete [3].

\section{Conclusion}

Acute pancreatitis may be complicated by venous thrombosis, most often the spleen vein's. The TMVS is rare, with vague clinical signs but easily detectable on CT scan. Anticoagulant therapy is effective if the diagnosis is made before the stage of bowel infarction. Its systematic implementation remains questionable in case of acute pancreatitis insomuch as spontaneous dissolution is possible.

\section{References}

[1] Na, B.S., John, B.M., Kim, K.B. and Lee, J.S. (2011) Spontaneous Dissolution of Isolated Superior Mesenteric Vein Thrombosis in Acute Pancreatitis. Korean Journal of Gastroenterology, 57, 38-41. http://dx.doi.org/10.4166/kjg.2011.57.1.38

[2] Morasch, M., Ebaugh, J., Chiou, A. and Matsumura, J. (2001) Mesenteric Venous Thrombosis: A Changing Clinical Entity. Journal of Vascular Surgery, 34, 680-684. http://dx.doi.org/10.1067/mva.2001.116965

[3] Rackoff, A., Shores, N. and Willner, I. (2005) Mesenteric Venous Thrombosis in a Patient with Pancreatitis and Protein C Deficiency. Southern Medical Journal, 98, 232-234. http://dx.doi.org/10.1097/01.SMJ.0000145305.95081.92

[4] Balthazar, E.L., Freeney, P.C. and van Sonnenberg, E. (1994) Imaging and Intervention in Acute Pancreatitis. Radiology, 193, 297-306. http://dx.doi.org/10.1148/radiology.193.2.7972730

[5] Crowe, P.M. and Sagar, G. (1995) Reversible Superior Mesenteric Vein Thrombosis in Acute Pancreatitis. Clinical Radiology, 50, 628-633. http://dx.doi.org/10.1016/S0009-9260(05)83293-3

[6] Mortelé, K.J., Mergo, P.J., Taylor, H.M. and Wiesner, W. (2004) Peripancreatic Vascular Abnormalities Complicating Acute Pancreatitis: Contrast-Enhanced Helical CT Findings. European Journal of Radiology, 52, 67-72. http://dx.doi.org/10.1016/j.ejrad.2003.10.006

[7] Cheung, D.Y., Kim, J.K., Jo, D.H. and Oh, H.J. (2005) A Case of Portal Vein Thrombosis Associated with Acute Pancreatitis and Cholangitis. Korean Journal of Gastroenterology, 46, 60-65. 